\title{
Presynaptic Dopamine Synthesis Capacity in Schizophrenia and Striatal Blood Flow Change During Antipsychotic Treatment and Medication-Free Conditions
}

\author{
Daniel Paul Eisenberg', Lisa Yankowitz', Angela M lanni', Dani Y Rubinstein', Philip D Kohn', \\ Catherine E Hegarty', Michael D Gregory', José A Apud' and Karen F Berman*,I \\ 'Section on Integrative Neuroimaging, Clinical \& Translational Neuroscience Branch, Intramural Research Program, National Institute of Mental \\ Health, National Institutes of Health, Department of Health and Human Services, Bethesda, MD, USA
}

\begin{abstract}
Standard-of-care biological treatment of schizophrenia remains dependent upon antipsychotic medications, which demonstrate $\mathrm{D}_{2}$ receptor affinity and elicit variable, partial clinical responses via neural mechanisms that are not entirely understood. In the striatum, where $\mathrm{D}_{2}$ receptors are abundant, antipsychotic medications may affect neural function in studies of animals, healthy volunteers, and patients, yet the relevance of this to pharmacotherapeutic actions remains unresolved. In this same brain region, some individuals with schizophrenia may demonstrate phenotypes consistent with exaggerated dopaminergic signaling, including alterations in dopamine synthesis capacity; however, the hypothesis that dopamine system characteristics underlie variance in medication-induced regional blood flow changes has not been directly tested. We therefore studied a cohort of 30 individuals with schizophrenia using longitudinal, multi-session [ ${ }^{15} \mathrm{O}$ ]-water and $\left[{ }^{18} \mathrm{~F}\right]-$ FDOPA positron emission tomography to determine striatal blood flow during active atypical antipsychotic medication treatment and after at least 3 weeks of placebo treatment, along with presynaptic dopamine synthesis capacity (ie, DOPA decarboxylase activity). Regional striatal blood flow was significantly higher during active treatment than during the placebo condition. Furthermore, medicationrelated increases in ventral striatal blood flow were associated with more robust amelioration of excited factor symptoms during active medication and with higher dopamine synthesis capacity. These data indicate that atypical medications enact measureable physiological alterations in limbic striatal circuitry that vary as a function of dopaminergic tone and may have relevance to aspects of therapeutic responses.
\end{abstract}

Neuropsychopharmacology (2017) 42, 2232-224I; doi:I0.I038/npp.2017.67; published online I0 May 20I7

\section{INTRODUCTION}

In schizophrenia, therapeutic trials of current pharmacotherapies have reported substantial rates of non- or partialresponses (Lieberman et al, 2005). The variability of response to treatment across individuals hampers confident prediction, lending a stochastic quality to clinical management of this debilitating illness. Despite small differences in their overall efficacy (Samara et al, 2016) and in their precise constellation of receptor affinities, currently available, standard-of-care, antipsychotic medications as a class continue to be unified by inhibition (antagonism or partial agonism) at the $\mathrm{D}_{2}$ dopamine receptor (Peroutka and Snyder, 1980; Seeman and Tallerico, 1999), although a definitive and detailed mechanistic understanding of their action in the brain has remained elusive.

*Correspondence: Dr KF Berman, Section on Integrative Neuroimaging, National Institute of Mental Health, NIH, Intramural Research Program, 9000 Rockville Pike, Building 10, Room 3Cl03A, Bethesda, MD 20892-I365, USA; Tel: + I 30| 496 7603, Fax: + I 30I 4967437, E-mail: Karen.Berman@nih.gov

Received 4 December 2016; revised 23 March 2017; accepted 24 March 2017; accepted article preview online 7 April 2017
To elucidate the neurophysiology of antipsychotic treatment response, several past imaging investigations have examined regional cerebral blood flow ( $\mathrm{rCBF}$ ) and metabolic changes induced by neuroleptics. Increases in regional cerebral blood flow in the striatum, where $\mathrm{D}_{2}$ receptors abound, have been observed after single doses of antipsychotic medication in healthy individuals measured with arterial spin labeling (Fernández-Seara et al, 2011; Handley et al, 2013; Viviani et al, 2013). Studies of individuals with schizophrenia have been less consistent; this same singledose effect has been observed in a positron emission tomography (PET) $\left[{ }^{15} \mathrm{O}\right.$-water rCBF study (Lahti et al, 2005), but other PET experiments have failed to find acute medication effects in patients (Goldman et al, 1996; Volkow et al, 1986). Whether individuals who are consistently treated with antipsychotic medications demonstrate persistently increased striatal activity has also been addressed by several studies, and increased striatal blood flow with treatment has been reported in longitudinal PET (Corson et al, 2002; Lahti et al, 2003, 2009b; Miller et al, 1997a) and SPECT (Livingston and the Scottish Schizophrenia Research Group, 1998; Miller et al, 1997b) studies, although, again, not all published data 
clearly corroborate these findings (Erkwoh et al, 1997; Ertugrul et al, 2009; Yildiz et al, 2000). Increases in striatal glucose metabolism have similarly been observed in several (Bartlett et al, 1991; Buchsbaum et al, 1987, 1992), although not all (Gur et al, 1987), reports. The suggestion that variability in antipsychotic medication-mediated functional changes in the basal ganglia may have important clinical implications has found initial explicit support from one study identifying increased ventral striatal rCBF measured with $\left[{ }^{15} \mathrm{O}\right]$-water PET after several weeks of antipsychotic treatment, relative to 2 weeks of medication withdrawal, an effect more frequently observed in individuals who responded to treatment (Lahti et al, 2009b).

Although a detailed mechanism to explain how antipsychotic medications might enact striatal blood flow changes has not been empirically demonstrated, a $\mathrm{D}_{2}$ dopamine receptor-driven model is plausible. Such a formulation is consistent with the considerable density of $\mathrm{D}_{2}$ receptors in this region and with the $\mathrm{D}_{2}$ receptor targeting common to all antipsychotic medications used in past experiments demonstrating this effect, including those studies employing firstgeneration agents (eg, haloperidol) with greater $\mathrm{D}_{2}$ receptor selectivity (Lahti et al, 2009a). $\mathrm{D}_{2}$ blockade is expected to result in disinhibition of indirect pathway medium spiny neuron (MSN) activity (Gerfen and Surmeier, 2011), consistent with early experiments demonstrating increased spontaneous striatal firing rates with 3 weeks of antipsychotic treatment (Skirboll and Bunney, 1979). Recent optogenetic-fMRI experimentation in mice has demonstrated that increasing the activity of $\mathrm{D}_{2}$-bearing $\mathrm{MSNs}$ alone can drive enhanced hemodynamic responses in the striatum (Lee et al, 2016), suggesting that antipsychoticinduced blood flow increases may be attributable to local MSN disinhibition. This effect may be particularly pronounced in individuals with greater presynaptic dopaminergic tone, and therefore greater dopaminergic modulation of MSN populations, at baseline. Alternative dopamine receptor blockade-related mechanisms-such as increased metabolic demands due to $\mathrm{D}_{2}$ receptor upregulation, indirect network effects via altered dopamine neuronal firing (Valenti et al, 2011), or disinhibition of corticostriatal glutamatergic inputs (Bamford et al, 2004)-may also play a role and may equally depend upon presynaptic dopamine system characteristics.

In both medicated and medication-free conditions, individuals suffering from schizophrenia on average, but not uniformly, demonstrate neurobiological phenotypes consistent with increased presynaptic dopaminergic function in the striatum. For instance, greater striatal dopamine release in response to amphetamine challenge (Abi-Dargham et al, 1998; Breier et al, 1997; Laruelle et al, 1996) and elevated striatal dopamine synthesis (Dao-Castellana et al, 1997; Hietala et al, 1995, 1999; Howes et al, 2009; Lindström et al, 1999; McGowan et al, 2004; Meyer-Lindenberg et al, 2002; Nozaki et al, 2009; Reith et al, 1994) have been well replicated neuroimaging observations in independent schizophrenia cohorts. There exists, however, considerable range and overlap with healthy individuals in these measurements, suggesting substantial variability in dopaminergic system characteristics among patients. It remains unproven whether such variability in presynaptic dopaminergic characteristics has any mechanistic relevance to individual differences in striatal functional responses to medication, despite prior suggestion that this may be the case (Lahti et al, 2009b). If so, then the degree to which an individual with primary psychosis expresses these dopaminergic phenotypes may be an important predictor of dopamine receptor-mediated neurophysiological changes with neuroleptic medications.

Hypothesizing not only that antipsychotic medication treatment would enhance striatal blood flow in a clinically meaningful manner, but also that greater presynaptic dopaminergic tone would correspond with greater blood flow changes, we studied a cohort of inpatients on the NIMH IRP schizophrenia ward who underwent a carefully observed cross-over, blinded, placebo-controlled antipsychotic medication withdrawal study with both $\left[{ }^{15} \mathrm{O}\right]$-water to measure rCBF (a gold standard indicator of regional metabolism (Fox et al, 1988)) and $\left[{ }^{18} \mathrm{~F}\right]$-fluorodopa PET to measure presynaptic dopamine synthesis and storage.

\section{MATERIALS AND METHODS}

\section{Participants}

Thirty volunteers between ages 18 and 59 (mean $27.7 \pm 8.2$ years; 9 women, 11 smokers) with schizophrenia (28) or schizoaffective disorder (2) participated after providing informed consent, as approved by the National Institutes of Health (NIH) Combined Neuroscience Institutional Review Board (IRB) and the NIH Radiation Safety Committee. Participants were recruited throughout the United States with IRB-approved advertisement activities. Diagnosis based on DSM-IV criteria was confirmed via clinicianadministered standardized clinical interview (SCID; First et al, 1996) and ongoing inpatient clinical evaluation. Patients were determined to be free of confounding psychiatric, neurological, substance-related, or major medical illness, as confirmed by structural magnetic resonance imaging (MRI), routine laboratory tests including urine toxicology, medical history, and physical examination. At the time of admission, all subjects were evaluated to be without a history of alcohol or substance addiction/dependence and were free of alcohol and substance abuse for a minimum of 3 months prior to the study. Four individuals completing all scans reported past alcohol abuse (none reported past daily use). Nine individuals in the full cohort (five in the multitracer group) reported past cannabis abuse (only three reported past daily use, although all had been abstinent for at least 2 years prior to admission, and frequency data were unavailable for one individual). All patients had a history of chronic illness and prior antipsychotic medication treatment exposure.

Each participant completed a dual-armed, balanced crossover, blinded medication withdrawal protocol, during which clinical assessments and PET scans occurred after at least 3 weeks of active antipsychotic treatment or medication withdrawal (17 placebo arm first; mean duration of placebo arm prior to $\left[{ }^{15} \mathrm{O}\right]$-water PET scan $27.8 \pm 2.1$ days). Antipsychotic medications (aripiprazole, olanzapine, quetiapine, risperidone, and ziprasidone) were administered in therapeutic doses, and chlorpromazine equivalent dose was calculated as previously described (Andreasen et al, 2010). Symptoms were assessed with the Positive and Negative Syndrome Scale (PANSS; Kay et al, 1987), as administered by 
blinded raters at both active medication and medication-free time points. Among factor analytic studies evaluating the structure of this instrument, there exists a reliable preponderance of similar five-factor solutions. We therefore adopted the consensus model approach established by Wallwork et al (2012), calculating the following symptom subscales: positive, negative, depressed, excited, and disorganized. Percent changes in symptoms were calculated as the medication-free rating minus the active medication rating, relative to the mean rating. This calculation was performed after rescaling the scores (subtracting one from each item) to correct for the fact that the absence of symptoms is rated 'one' (rather than 'zero') for each PANSS item, as previously discussed (Obermeier et al, 2010).

\section{Neuroimaging}

Acquisition procedures. PET measurement of rCBF was conducted twice-once during placebo and once during the active treatment arm-with $\left[{ }^{15} \mathrm{O}\right]$-water PET in all 30 participants. In a separate PET session during the placebo arm, presynaptic dopamine synthesis capacity was measured with $\left[{ }^{18} \mathrm{~F}\right]-$ FDOPA in 18 of the 30 participants. In addition, structural MRI at $3 \mathrm{~T}$ was acquired for each subject separately to generate a T1-weighted anatomical image to guide PET data preparation as described below.

Caffeine and nicotine were not permitted for $4 \mathrm{~h}$ before all scanning sessions. In addition, prior to $\left[{ }^{18} \mathrm{~F}\right]$-FDOPA PET sessions, a fasting state (minimum $6 \mathrm{~h}$ ) was required to avoid competition for tracer central nervous system (CNS) admittance via the L-type large neutral amino-acid carrier system. Oral administration of $200 \mathrm{mg}$ of carbidopa, a peripheral amino-acid decarboxylase inhibitor, was given $1 \mathrm{~h}$ prior to $\left[{ }^{18} \mathrm{~F}\right]-F D O P A$ injection to limit peripheral metabolism of circulating radiotracer and, thus, maximize its availability to the CNS.

All PET neuroimaging was performed with a General Electric Advance 3D PET camera (32 planes, $6.5 \mathrm{~mm}$ FWHM). To limit head motion during data acquisition, an individually molded thermoplastic mask was applied. An 8-min transmission scan for attenuation correction was collected for all PET scan sessions. For the resting-state $\left[{ }^{15} \mathrm{O}\right]$-water data, two 60-s long emission scans were collected after intravenous administration of $10 \mathrm{mCi}(370 \mathrm{MBq})$ of $\left[{ }^{15} \mathrm{O}\right]$-water with an interscan interval of $6 \mathrm{~min}$. For the $\left[{ }^{18} \mathrm{~F}\right]$-FDOPA data, 27 dynamically acquired emission scan frames were collected over 90 min after intravenous administration of $\sim 16 \mathrm{mCi}(592 \mathrm{MBq})$ of $\left[{ }^{18} \mathrm{~F}\right]$-FDOPA.

T1-weighted structural MRI scans were acquired separately on a $3 \mathrm{~T}$ General Electric scanner for coregistration and warping, as described below.

Data processing procedures. For all PET scans, filtered back-projection reconstruction using the $3 \mathrm{D}$ reprojection method (Kinahan and Rogers, 1989) and employing a Hanning filter was performed with corrections for postinjection radioactivity decay, dead time, and scatter; and a registered attenuation correction algorithm that accounted for frame-wise head motion was applied.

T1-weighted anatomical MRI images were nonparametric nonuniform intensity normalized (Sled et al, 1998) and aligned to the anterior-posterior commissure. Using an initial

segmentation of these images by Freesurfer (http://freesurfer. net/) as a starting point, bilateral striatal regions of interest (ROIs) - caudate, putamen, and ventral striatum (defined as the nucleus accumbens and adjacent striatal tissue inferior to the anterior commissure)-were manually drawn for each individual by operators blinded to the PET data (Yushkevich et al, 2006) and eroded by one voxel to mitigate partial volume effects. For use as a reference region for kinetic analyses of the $\left[{ }^{18} \mathrm{~F}\right]$-FDOPA data, a gray matter cerebellar reference region (excluding vermis and lateral/superior parasinus regions) was also drawn for each individual.

For the $\left[{ }^{15} \mathrm{O}\right]$-water data, a correction for background activity was performed on each scan, and the two scans obtained for each PET session were aligned and global signal normalized to account for variation across injections in overall activity counts. The resulting image pairs were averaged within each session. Each individual's T1-weighted anatomical MRI image was coregistered to his or her mean PET image along with the corresponding segmentation image generated from the ROI derivation described above. Mean striatal ROI values were then extracted. Blood flow change was calculated via simple subtraction (active-placebo) for each region. For voxel-wise analyses, the coregistered anatomical MRI image was warped to a common-space (MNI) template using ANTS software (http://stnava.github.io/ANTs/). The resulting transformation matrix was applied to the PET images. Blood flow change maps were generated by subtracting the medicationfree mean rCBF maps from those of the active medication arm.

For the $\left[{ }^{18} \mathrm{~F}\right]$-FDOPA data, the 4 th through 27 th emission frames were corrected for interscan motion by realignment to the 21st frame with a rigid body transformation using FLIRT (http://fsl.fmrib.ox.ac.uk/fsl/); the first three frames, which have lower signal to guide realignment, used the transform of the fourth frame. The T1-weighted anatomical MRI image for each subject was coregistered to the mean of his or her PET images using SPM5 (http://www.fil.ion.ucl.ac. $\mathrm{uk} / \mathrm{spm} /$ ). Striatal ROI and cerebellar reference region timeactivity curves were extracted from the emission frames, and the kinetic rate constant, $K_{\mathrm{i}}$, representing specific tracer uptake and a measurement of dopamine synthesis capacity (DOPA decarboxylase activity), was calculated for each ROI with PMOD software (http://www.pmod.com/), using the non-invasive graphical linearization method established by Patlak and Blasberg (1985).

\section{Statistical Analyses}

Statistical analyses for demographic, clinical, and striatal ROI association testing were performed in SPSS (http://www-01. $\mathrm{ibm} . \mathrm{com} /$ software/analytics/spss/) with general linear modeling and, for analyses with PANSS scores, nonparametric tests. For the latter, differences across medication conditions were tested with related-samples Wilcoxon signed-rank tests. Associations between PANSS change scores and binary or continuous variables were tested with independent samples Mann-Whitney U-tests and Spearman's rank correlations, respectively. For tests of association between striatal PET measurements and patient characteristics, multivariate tests were conducted with the three bilateral ROI rCBF change values or $K_{\mathrm{i}}$ values as dependent variables, and significant results were followed up with post hoc univariate testing. For pairwise tests of association between each ROI rCBF change 
Table I Clinical Ratings Data

\begin{tabular}{|c|c|c|c|c|c|c|c|c|}
\hline Group & $N$ & $\begin{array}{l}\text { PANSS } \\
\text { measure }\end{array}$ & Total & Depressed & Disorganized & Excited & Negative & Positive \\
\hline \multirow[t]{2}{*}{ Full } & 30 & Active & $60(55$ to 72.8$)$ & 6.5 (5 to 8$)$ & $6(5$ to 8.3$)$ & $5(4$ to 5.3$)$ & I 6.5 (1 | .8 to 21$)$ & $8(7$ to 11$)$ \\
\hline & & $\begin{array}{l}\text { Percent } \\
\text { change }\end{array}$ & $9.4(-17.4$ to 36.9$)$ & 9.1 ( -57.6 to 60.9$)$ & | $6.8(-3 \mid .4$ to 81.4$)$ & $0(-46.7$ to 75$)$ & $0(-25.4$ to 23.4$)$ & I $8.2(-35$ to 54.5$) \dagger$ \\
\hline & & $\begin{array}{l}\text { Percent } \\
\text { change }\end{array}$ & $20.9(-11.3 \text { to } 36.9)^{*}$ & $18.2(-40$ to 60.9$)$ & $21.6(-26.7$ to 71.4$)$ & $0(-55$ to 200$)$ & $6.7(-25$ to 68.4$)$ & $23.4(-19.9$ to 50$) *$ \\
\hline
\end{tabular}

For the full patient cohort who completed two regional cerebral blood flow (rCBF) scans, and for those who completed $\left[{ }^{18} \mathrm{~F}\right]$-FDOPA PET scans as well as the rCBF assessments, PANSS ratings median values and interquartile ranges are provided. Asterisks indicate a significant effect of condition at $p<0.05$. Daggers indicate nonsignificant trend $(p<0.1)$.

and their respective $\left[{ }^{18} \mathrm{~F}\right]$-FDOPA $K_{\mathrm{i}}$ measurement, simple univariate tests were conducted. For finer anatomical localization of significant PET results, voxel-wise general linear model analyses of $\mathrm{rCBF}$ change were conducted in SPM (http://www.fil.ion.ucl.ac.uk/spm/) within the striatum with an uncorrected voxel-wise threshold of $p<0.005$. These included association tests with $K_{\mathrm{i}}$ values and PANSS change scores. The latter were rank-transformed prior to entrance in voxel-wise analyses.

\section{RESULTS}

\section{Demographics and Clinical Characteristics}

Patients had been ill for an average of $6.6( \pm 6.0)$ years prior to participation, and received standard doses of antipsychotic medication during the active arm of the protocol (mean chlorpromazine equivalent dose $303.3 \pm 102.5 \mathrm{mg}$ ). The subsample who completed $\left[{ }^{18} \mathrm{~F}\right]$-FDOPA PET scanning (injected dose $595.3 \pm 28.8 \mathrm{MBq}$, specific activity $57.2 \pm 18.3 \mathrm{MBq} / \mu \mathrm{mol}$, mass $2.5 \pm 0.9 \mathrm{mg}$ ) shared similar demographics as the full sample (18 subjects, all with a diagnosis of schizophrenia, 9 female, age $29.3 \pm 10.2$ years, 5 smokers, duration of illness $7.8 \pm 7.3$ years, chlorpromazine equivalent dose $278.9 \pm 83.9 \mathrm{mg}$, 9 started with the placebo arm). PANSS ratings indicated symptoms in the mild-tomoderate range with considerable interindividual variation in baseline and response ratings. Although decompensation during medication withdrawal was not seen by this rating scale in all patients, in the full group, there was a trend for greater positive symptoms during the placebo arm $(z=1.77$, $p=0.077$ ). In the multi-tracer group (those who completed the $\left[{ }^{18} \mathrm{~F}\right]$-FDOPA scans as well as the two rCBF scans), a significant effect of medication condition was observed, with greater total and positive symptom burden during the medication-free period (total: $z=2.03, p=0.043$; positive: $z=2.27, p=0.023)$. In this group, a non-significant trend for greater disorganized symptoms was also noted $(z=1.65$, $p=0.099$ ). See Table 1 .

Percent change in clinical ratings, as measured by the PANSS total or subscale scores, did not show any significant relationship with sex, chlorpromazine equivalent dose, or the order of the treatment arms in the full group or the subgroup. In the full group only, smoking was associated with total and excited symptom change (total: $U=53.5$, $p=0.026$; excited: $U=45, p=0.009$ ), such that non-smokers tended to show greater decompensation off of medication, and we therefore repeated PANSS association tests in this cohort controlling for smoking. In the multi-tracer subgroup, there was no relationship between smoking and PANSS score changes. In the multi-tracer but not the full group, older age was associated with greater disorganized symptoms increase during the placebo arm $(\rho=0.56, p=0.016)$.

\section{Neuroimaging}

$r C B F$ change with antipsychotic medication treatment. During active treatment relative to placebo, all bilateral subregions of the striatum had greater rCBF, particularly in the ventral striatum (full group: putamen $t(29)=2.65$, $p=0.013$; caudate $t(29)=2.41, p=0.023$; ventral striatum $t(29)=2.99, \quad p=0.006 ; \quad$ multi-tracer group: putamen $t(17)=2.43, p=0.026$; caudate $t(17)=2.46, p=0.025$; ventral striatum $t(17)=3.61, p=0.002)$. These findings remained significant after repeating analyses without the two individuals with schizoaffective disorder and were corroborated further by post hoc voxel-wise tests (peak $p=0.0001$, uncorrected; $p=0.017$, FDR corrected; see Table 2; Figure 1).

rCBF change and patient characteristics. Age did not show significant relationships with striatal $\mathrm{rCBF}$ percent change in the full group, but in the multi-tracer group, a trend existed (multivariate Pillai's Trace $\mathrm{F}(3,14)=2.74$, $p=0.083$ ). Follow-up univariate testing suggested a trendlevel association with age in the putamen $(\mathrm{F}(1,16)=3.94$, $p=0.065)$ and a significant relationship in the caudate $(\mathrm{F}(1,16)=4.94, p=0.041)$ where age was inversely related to medication-induced striatal $\mathrm{rCBF}$ enhancement. To rule out confounding age effects, we therefore repeated $\mathrm{rCBF}$ analyses described below for this group statistically controlling for age. Multivariate tests did not find significant relationships between striatal rCBF change and smoking, sex, chlorpromazine equivalent dose, or treatment-arm order in either group.

In the full group, there was no association between striatal rCBF change and total PANSS score change; however, percent change in the excited factor score predicted striatal rCBF change (putamen: $\rho=0.45, p=0.012$; caudate: $\rho=0.33$, 
Table 2 Voxel-wise Analyses Results

\begin{tabular}{|c|c|c|c|c|c|c|c|c|c|}
\hline Effect & \multicolumn{2}{|c|}{ Region } & $x$ & $Y$ & $\mathbf{Z}$ & Size (voxels) & T-value & $p$-value (FDR) & $p$-value \\
\hline \multicolumn{10}{|c|}{ Effect of medication } \\
\hline \multicolumn{10}{|c|}{ rCBF change (active-placebo) } \\
\hline & $L$ & Ventral striatum & -31.5 & -6 & -7.5 & 632 & 4.25 & 0.017 & 0.00010 \\
\hline & $\mathrm{R}$ & Caudate & 12 & 3 & 9 & 186 & 4.10 & 0.017 & 0.00015 \\
\hline & $\mathrm{R}$ & Ventral striatum & 27 & 7.5 & -9 & 465 & 3.85 & 0.017 & 0.00030 \\
\hline & $\mathrm{R}$ & Putamen & 33 & -9 & -1.5 & 93 & 3.75 & 0.017 & 0.00039 \\
\hline & $L$ & Putamen & -19.5 & 19.5 & -9 & 4 & 2.80 & 0.020 & 0.0045 \\
\hline \multicolumn{10}{|c|}{ Effect of symptom change } \\
\hline \multicolumn{10}{|c|}{$\begin{array}{l}\text { rCBF (active_placebo) association } \\
\text { with excited factor score } \\
\text { percent change } \\
\text { (placebo_active) rank }\end{array}$} \\
\hline & $\mathrm{R}$ & Ventral striatum & 24 & 13.5 & -15 & 1202 & 5.17 & 0.0040 & $8.64 \mathrm{E}-06$ \\
\hline & $\mathrm{R}$ & Ventral striatum & 24 & 13.5 & -15 & 1202 & 5.17 & 0.0040 & $8.64 \mathrm{E}-06$ \\
\hline & $L$ & Putamen & -24 & 18 & 1.5 & 208 & 4.22 & 0.0040 & $1.15 E-04$ \\
\hline & $L$ & Caudate & -19.5 & 19.5 & 1.5 & 7 & 3.08 & 0.012 & 0.0023 \\
\hline \multicolumn{10}{|c|}{ Effect of dopamine synthesis } \\
\hline \multicolumn{10}{|c|}{$\begin{array}{l}\text { rCBF percent change (active_-placebo) } \\
\text { association with ventral } \\
\text { striatal dopamine synthesis } \\
\text { capacity }\end{array}$} \\
\hline & $L$ & Ventral striatum & -15 & 15 & -12 & 273 & 4.21 & 0.078 & 0.00033 \\
\hline
\end{tabular}

Effects of medication and symptom change reported for the full patient cohort who completed two regional cerebral blood flow (rCBF) scans. Effect of dopamine synthesis is reported for the subset of those who also completed $\left[{ }^{18} \mathrm{~F}\right]$-FDOPA PET scans. FDR correction was applied voxel-wise for the striatal search volume.

$p=0.071$; ventral striatum: $p=0.44, p=0.014$; see Figure 2). This result indicated that individuals whose excited symptoms improved most on medication also showed greater rCBF increases on medication, an effect that remained significant when the two individuals with schizoaffective disorder were removed or when partial nonparametric correlations were performed controlling for age, sex, chlorpromazine equivalent dose, or treatment-arm order. When controlling for smoking, only the putamen association remained significant. No other subscales showed any significant associations with blood flow alterations. A voxel-wise analysis of association between $\mathrm{rCBF}$ change and ranked change in excited subscale scores revealed corresponding effects in bilateral striatal loci, which were particularly strong in the right subcommissural striatum, including the ventral putamen (peak $p=8.6 \times 10^{-6}$, uncorrected; $p=0.004$, FDR corrected; see Table 2; Figure 2).

Presynaptic dopamine synthesis capacity and patient characteristics. Multivariate testing showed no associations between striatal $\left[{ }^{18} \mathrm{~F}\right]$-FDOPA-specific uptake $\left(K_{\mathrm{i}}\right)$, a measure of presynaptic dopamine synthesis capacity, and age, sex, smoking status, chlorpromazine equivalent dose, treatment-arm order, $\left[{ }^{18} \mathrm{~F}\right]-$ FDOPA dose, $\left[{ }^{18} \mathrm{~F}\right]-\mathrm{FDOPA}-$ specific activity, $\left[{ }^{18} \mathrm{~F}\right]-$ FDOPA mass, past alcohol use, or past cannabis use. Spearman's rank correlations revealed an association between positive symptom increases off of medication and lower caudate $K_{\mathrm{i}}(\rho=-0.49, p=0.037)$, with a trend for the same effect in the putamen $(\rho=-0.40$, $p=0.097)$. This was retained in partial correlation analyses controlling for age, chlorpromazine equivalents, or smoking status, but only a trend-level association remained in the caudate when controlling for sex or treatment-arm order.

rCBF change and presynaptic dopamine synthesis capacity. Ventral striatal $\left[{ }^{18} \mathrm{~F}\right]-$ FDOPA $K_{\mathrm{i}}$ predicted rCBF change in the ventral striatum $(\mathrm{F}(1,16)=8.018, p=0.012)$, but putamen and caudate $K_{\mathrm{i}}$ did not predict putamen or caudate rCBF change, respectively (Figure 3 ). This relationship remained significant when including age, sex, smoking status, chlorpromazine equivalent dose, or treatment-arm order in the model. Voxelwise examination of this association localized the strongest effect to a prominent cluster abutting the left nucleus accumbens anterosuperiorly (peak $p=0.00033$, uncorrected; $p=0.078$, FDR corrected; see Table 2; Figure 3). For all three striatal subregions, there was not a significant correlation of $\left[{ }^{18} \mathrm{~F}\right]-$ FDOPA $K_{\mathrm{i}}$ and either placebo or active treatment rCBF alone (Supplementary Figures 1 and 2).

\section{DISCUSSION}

Individuals with schizophrenia showed increased striatal rCBF after at least 3 weeks of stable antipsychotic pharmacotherapy, relative to placebo treatment, in line with prior work 

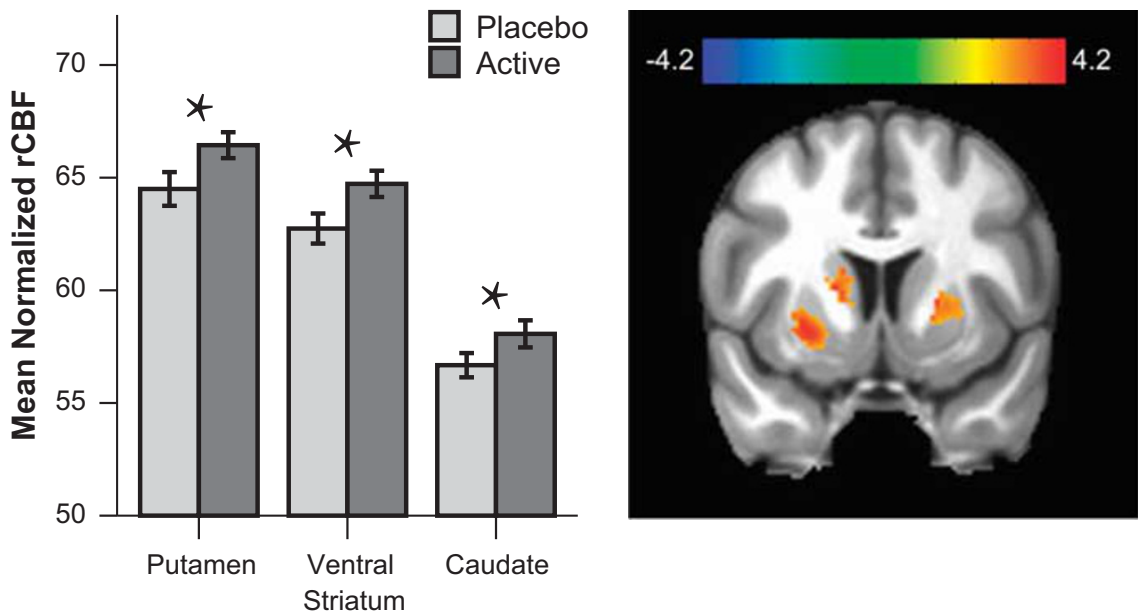

Figure I Antipsychotic medication effects on regional cerebral blood flow. The graph shows mean regional cerebral blood flow ( $r$ CBF) for each bilateral region of interest $(\mathrm{ROI})$, with SE bars and asterisks indicating significance at $p<0.05$. The coronal slice (MNI $y=9$ mm) shows voxel-wise analysis results indicating locations of greatest medication effects in the striatum. Colored clusters represent areas where rCBF was greater during active medication relative to placebo. The color bar indicates T-values, and data are displayed by radiological convention (right hemisphere on the left side) at a voxel-wise, uncorrected threshold of $p<0.005$.
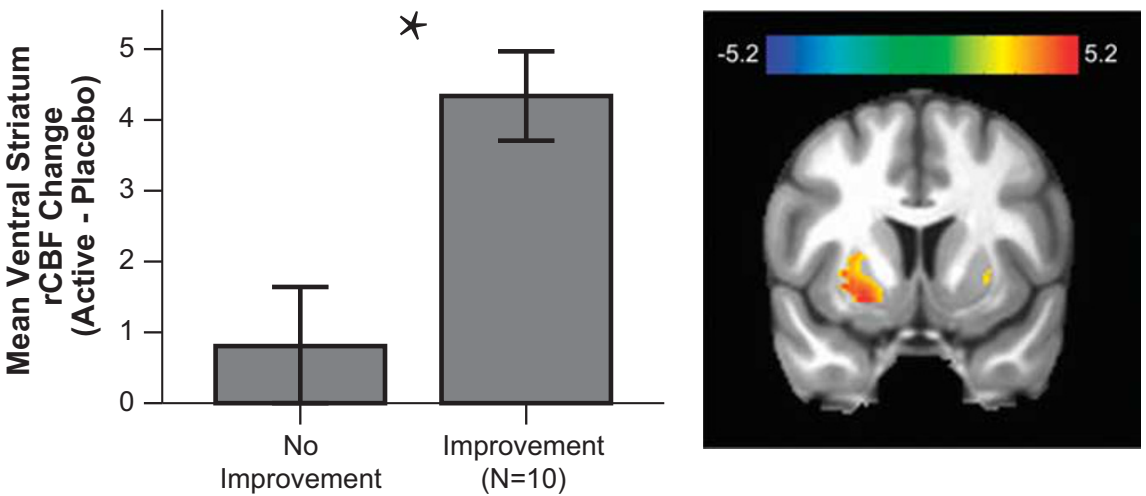

$(\mathrm{N}=20)$

Excited Symptom Change
with Active Treatment

Figure 2 Relationship between regional cerebral blood flow (rCBF) change and symptoms. Mean ventral striatal medication-induced rCBF increases and SE bars are shown for the full cohort, categorized by whether PANSS-excited symptom scores improved during active medication treatment (ie, percent change $>0$ ) or not (ie, percent change $\leqslant 0$ ), with significance at $p<0.05$ indicated by the asterisk. The coronal slice at MNI $y=9 \mathrm{~mm}$ shows results of voxel-wise analyses indicating locations of greatest correlation between ranked symptom change and blood flow change in the striatum. Colored clusters represent areas where medication-induced increases in rCBF correspond to medication-induced improvements in excited factor symptoms. Color bars indicate T-values, and data are displayed by radiological convention at a voxel-wise, uncorrected threshold of $p<0.005$.

(Lahti et al, 2009b). This finding is concordant with several single-dose studies in healthy individuals and patients (Fernández-Seara et al, 2011; Handley et al, 2013; Lahti et al, 2005; Viviani et al, 2013), suggesting both that neurolepticinduced basal ganglia blood flow increases are a replicable observation even in the course of several weeks of treatment and, by the same token, that such changes are not unalterable, as, on average, they were decreased with medication withdrawal. Given the close association between regional neural activity and blood flow (Fox et al, 1988; Sokoloff, 1961), these findings indicate that local striatal neural operations increase with this pharmacological treatment. Although direct vascular modulation by antipsychotics at smooth muscle receptor sites cannot be ruled out and may merit future study (AfonsoOramas et al, 2014; Krimer et al, 1998), such an effect is unlikely to fully explain the observed results, given the sheer density of neuronal dopaminergic terminals in this region and the fact that the current method controls for global intracerebral counts, and therefore for general neurovascular effects. The rCBF increase with neuroleptic treatment, although present diffusely throughout the striatum, was most robust in the ventral striatum, a central node in mesolimbic systems implicated under some models of schizophrenia and neuroleptic action (Grace, 2015; Lahti et al, 2003).

In agreement with past studies by Lahti et al, 2009b, we found that greater striatal $\mathrm{rCBF}$ increase was associated with greater improvements of some symptoms on active medication relative to placebo. Although total PANSS ratings did not show this relationship, the excited factor subscale did. This factor represents a group of symptoms that previously 


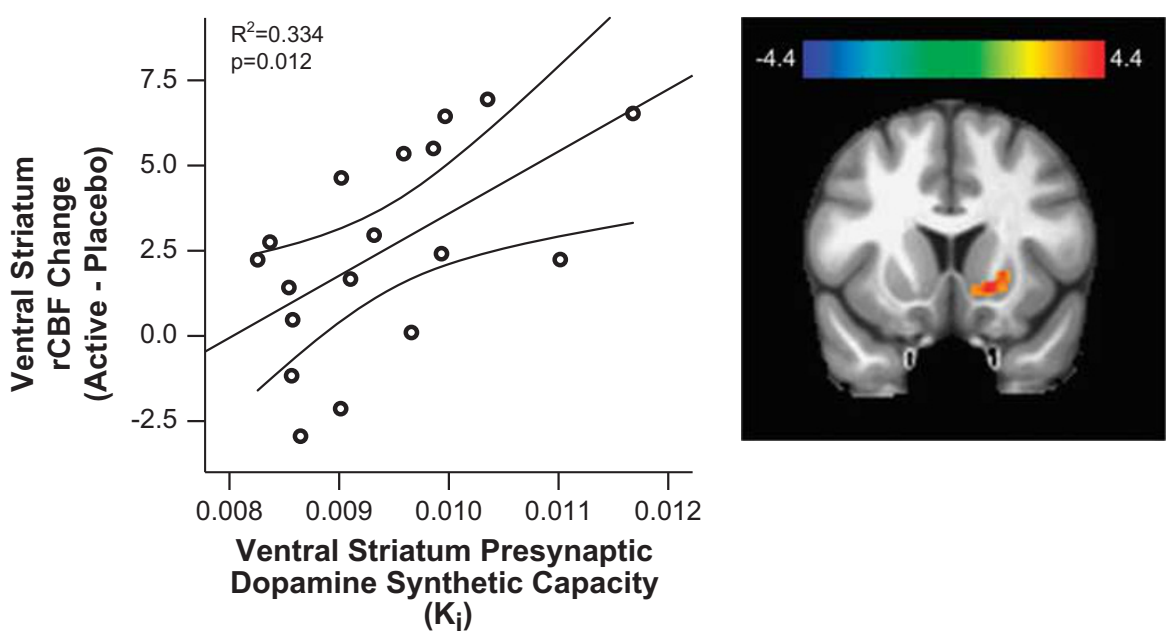

Figure 3 Relationship between regional cerebral blood flow change ( $\mathrm{rCBF}$ ) and dopamine synthesis capacity. The plot indicates ventral striatal rCBF change as a function of ventral striatal $\left.{ }^{18} \mathrm{~F}\right]$-FDOPA-specific uptake $\left(K_{i}\right)$. The regression line (intercept: -14.64 ; slope: $\left.1.82 \times 10^{3} ; R^{2}=0.334, R=0.578\right)$ and $95 \%$ confidence limits are shown. The coronal slice at MNI $y=15 \mathrm{~mm}$ shows results of voxel-wise analyses indicating locations of greatest positive association between ventral striatal presynaptic dopamine synthesis capacity $\left(K_{i}\right)$ and striatal rCBF change (active-placebo). Color bars indicate T-values, and data are displayed by radiological convention at a voxel-wise, uncorrected threshold of $p<0.005$.

have shown responsiveness to antipsychotic medications (Victoroff et al, 2014) and includes excitement, hostility, uncooperativeness, and impulsivity items (Wallwork et al, 2012). Over the duration of our study, a third of our sample demonstrated improvements on this measure with antipsychotic treatment. Therapeutic response based on the standard positive symptom subscale of the PANSS (which includes two excitement factor items), but not the standard negative symptom subscale (which includes no excitement factor items), has been associated with striatal, but not extrastriatal, $\mathrm{D}_{2}$ antipsychotic occupancy, reaffirming the clinical importance of understanding striatal dopamine systems in this context (Agid et al, 2007).

It is clear from the schizophrenia literature that there exists a profound heterogeneity of both clinical illness and underlying neurobiology of individuals with schizophrenia. To the extent that illness heterogeneity is a major barrier for the development of generalizable knowledge and novel treatment approaches in schizophrenia, understanding the biological foundations of such variability is essential. As a prime example, past work has shown striatal presynaptic dopamine synthesis capacity to be abnormal to varying degrees across schizophrenia patients, suggesting a broad range of dopaminergic systems functioning in this illness, which may have important therapeutic implications (Dao-Castellana et al, 1997; Hietala et al, 1995, 1999; Howes et al, 2009; Lindström et al, 1999; McGowan et al, 2004; Meyer-Lindenberg et al, 2002; Nozaki et al, 2009; Reith et al, 1994). Similar to these studies, we found that there was substantial variability in medication-induced striatal blood flow change across the present cohort. Although unmeasured aspects of pharmaceutical kinetics and metabolism cannot be ruled out as contributory, there was insufficient evidence to support the claim that this variability was driven solely by the strength of administered $\mathrm{D}_{2}$ antagonism, given the lack of association between this treatment effect and chlorpromazine equivalent dose.

Hypothesizing that tonic dopaminergic system characteristics may be related to the magnitude of $\mathrm{rCBF}$ response to neuroleptics relative to the medication-free state, we tested for association between dopamine synthesis capacity (FDOPA uptake) and antipsychotic-induced rCBF change. Importantly, and in accord with this proposal, in the subcommissural striatum, where the most significant antipsychotic medication-induced $\mathrm{rCBF}$ change was observed, greater presynaptic dopamine synthesis capacity predicted greater blood flow increase with medications. Thus, those individuals with higher dopamine tone-and therefore greater expression of this illness-associated phenotype-demonstrated a more vigorous neurophysiological response to medication. In line with past work showing enhanced striatal activity with typical antipsychotic medications (Corson et al, 2002; Lahti et al, 2003, 2009b; Miller et al, 1997a), this finding suggests a dopamine-related mechanism underlying the striatal rCBF increase observed here. It is possible that greater tonic availability of dopamine associated with enhanced DOPA decarboxylase (DDC) activity, or greater quantities of DDC containing terminals, results in greater neural activity changes when dopaminergic blockade is enacted (ie, greater 'gain'); however, this speculation requires confirmation from future investigations. With respect to the subregional localization of our findings, although recent literature has highlighted the importance of more dorsal striatal regions in schizophrenia pathophysiology (Weinstein et al, 2017), evidence has also suggested that ventral striatal regions may be preferentially sensitive to some types of dopaminergic pharmacological interventions such as amphetamine administration (Martinez et al, 2003) and atypical antipsychotic medication treatment (Lahti et al, 2009b). Continued experimentation in larger samples is indicated to better characterize potentially important functional and neurochemical distinctions between dorsal and ventral striatal circuitry relevant both to schizophrenia and neuroleptic action. Nonetheless, our data suggest that at least some of the substantial individual variation in striatal presynaptic dopaminergic tone that has been reported over the past two decades is linked to treatment-relevant neurophysiology. 
This work is subject to several caveats and limitations. The use of several different atypical antipsychotic agents in this work ensured that participants would be able to be studied under optimal, individualized therapeutic medication conditions, thereby minimizing confounds of medication intolerances and inefficacy. However, the varied agents used (and/or other potential confounds) may have added noise to the reported relationships, and future studies with larger sample sizes, powered not only to confirm the present findings but also to examine possible differences in observed effects across pharmacological agents will be even more informative. A number of uncontrolled variables could have contributed to the present findings, including hormone/menstrual status (Berman et al, 1997), sleep quality/arousal (Braun et al, 1997), and the nature of individual thought processes during restingstate rCBF acquisition (Sokoloff, 1961). However, the withinsubject design, mean interscan interval of $\sim 1$ month, and consistency of medication effects with prior work offer some reassurance with respect to these concerns. The placebocontrolled, counterbalanced design carried out on the NIMH IRP inpatient ward with close clinical and medication adherence monitoring is also a strength. Although the mean ventral striatum $K_{\mathrm{i}}$ values reported here are comparable to those of healthy individuals studied elsewhere with the same scanner model (Jokinen et al, 2009), methodological differences require caution when comparing the present data and those published from other PET centers. In addition, future work comparing patients and matched healthy individuals on these measurements will be even more informative. Finally, we note that the preponderance of dopamine terminals in the striatum provides reasonable support for the interpretation of the striatal $\left[{ }^{18} \mathrm{~F}\right]$-FDOPA signal as dopaminergic; however, we cannot exclude the possibility that other monoaminergic (eg, serotonergic) terminals that may also express DDC, contribute to the observed signal.

In conclusion, we provide confirmatory evidence for increase of striatal blood flow during antipsychotic treatment relative to medication-free states, even when both treatment and medication-free conditions are established for several weeks. This increase may be clinically relevant, given its association with excitement factor symptoms. Furthermore, its association with dopamine synthesis capacity in the ventral regions of the striatum bolsters support for a neurochemical mechanism underpinning its interindividual variability and brings together two long-standing neuroimaging phenotypes in schizophrenia.

\section{FUNDING AND DISCLOSURE}

This research was supported by the Intramural Research Program, National Institute of Mental Health, National Institutes of Health, Bethesda, MD, USA. Project ZIAMH002652/NCT00001247/89-M-0160. The authors declare no conflict of interest.

\section{ACKNOWLEDGMENTS}

We thank the staffs of the NIH PET Center and the NIMH Intramural Research Program Inpatient Ward for their assistance in data acquisition and patient care.

\section{REFERENCES}

Abi-Dargham A, Gil R, Krystal J, Baldwin RM, Seibyl JP, Bowers M et al (1998). Increased striatal dopamine transmission in schizophrenia: confirmation in a second cohort. Am J Psychiatry 155: 761-767.

Afonso-Oramas D, Cruz-Muros I, Castro-Hernández J, Salas-Hernández J, Barroso-Chinea P, García-Hernández S et al (2014). Striatal vessels receive phosphorylated tyrosine hydroxylase-rich innervation from midbrain dopaminergic neurons. Front Neuroanat 8: 84.

Agid O, Mamo D, Ginovart N, Vitcu I, Wilson AA, Zipursky RB et al (2007). Striatal vs extrastriatal dopamine D2 receptors in antipsychotic response-a double-blind PET study in schizophrenia. Neuropsychopharmacology 32: 1209-1215.

Andreasen NC, Pressler M, Nopoulos P, Miller D, Ho B-C (2010). Antipsychotic dose equivalents and dose-years: a standardized method for comparing exposure to different drugs. Biol Psychiatry 67: 255-262.

Bamford NS, Zhang H, Schmitz Y, Wu N-P, Cepeda C, Levine MS et al (2004). Heterosynaptic dopamine neurotransmission selects sets of corticostriatal terminals. Neuron 42: 653-663.

Bartlett EJ, Wolkin A, Brodie JD, Laska EM, Wolf AP, Sanfilipo M (1991). Importance of pharmacologic control in PET studies: effects of thiothixene and haloperidol on cerebral glucose utilization in chronic schizophrenia. Psychiatry Res 40: 115-124.

Berman KF, Schmidt PJ, Rubinow DR, Danaceau MA, Van Horn JD, Esposito G et al (1997). Modulation of cognition-specific cortical activity by gonadal steroids: a positron-emission tomography study in women. Proc Natl Acad Sci USA 94: 8836-8841.

Braun AR, Balkin TJ, Wesenten NJ, Carson RE, Varga M, Baldwin P et al (1997). Regional cerebral blood flow throughout the sleep-wake cycle. An H2(15)O PET study. Brain 120(Pt 7): 1173-1197.

Breier A, Su T-P, Saunders R, Carson RE, Kolachana BS, de Bartolomeis A et al (1997). Schizophrenia is associated with elevated amphetamine-induced synaptic dopamine concentrations: Evidence from a novel positron emission tomography z_nbsp;method. Proc Natl Acad Sci USA 94: 2569-2574.

Buchsbaum MS, Potkin SG, Siegel BV Jr, Lohr J, Katz M, Gottschalk LA et al (1992). Striatal metabolic rate and clinical response to neuroleptics in schizophrenia. Arch Gen Psychiatry 49: 966-974.

Buchsbaum MS, Wu JC, DeLisi LE, Holcomb HH, Hazlett E, Cooper-Langston K et al (1987). Positron emission tomography studies of basal ganglia and somatosensory cortex neuroleptic drug effects: Differences between normal controls and schizophrenic patients. Biol Psychiatry 22: 479-494.

Corson PW, O'Leary DS, Miller DD, Andreasen NC (2002). The effects of neuroleptic medications on basal ganglia blood flow in schizophreniform disorders: a comparison between the neuroleptic-naïve and medicated states. Biol Psychiatry 52: 855-862.

Dao-Castellana M-H, Paillère-Martinot M-L, Hantraye $P$, Attar-Lévy D, Rémy P, Crouzel C et al (1997). Presynaptic dopaminergic function in the striatum of schizophrenic patients. Schizophr Res 23: 167-174.

Erkwoh R, Sabri O, Steinmeyer EM, Büll U, Saß H (1997). Psychopathological and SPECT findings in never-treated schizophrenia. Acta Psychiatr Scand 96: 51-57.

Ertugrul A, Volkan-Salanci B, Basar K, Karli Oguz K, Demir B, Ergun EL et al (2009). The effect of clozapine on regional cerebral blood flow and brain metabolite ratios in schizophrenia: Relationship with treatment response. Psychiatry Res 174: 121-129.

Fernández-Seara MA, Aznárez-Sanado M, Mengual E, Irigoyen J, Heukamp F, Pastor MA (2011). Effects on resting cerebral blood flow and functional connectivity induced by metoclopramide: a 
perfusion MRI study in healthy volunteers. Br J Pharmacol 163: 1639-1652.

First MB, Gibbon M, Spitzer RL, Williams JBW (1996). User's Guide for the SCID-I for DSM-IV Axis I Disorders - Research Version. Biometrics Research: New York.

Fox PT, Raichle ME, Mintun MA, Dence C (1988). Nonoxidative glucose consumption during focal physiologic neural activity. Science 241: 462.

Gerfen CR, Surmeier DJ (2011). Modulation of striatal projection systems by dopamine. Annu Rev Neurosci 34: 441-466.

Goldman RG, Alexander GE, Zemishlany Z, Mukherjee S, Sackeim H, Prohovnik I (1996). Acute effects of haloperidol on cerebral cortex blood flow in normal and schizophrenic subjects. Biol Psychiatry 40: 604-608.

Grace AA (2015). Dopamine system dysregulation and the pathophysiology of schizophrenia: insights from the methylazoxymethanol acetate model. Biol Psychiatry 81: 5-8.

Gur RE, Resnick SM, Gur RC, Alavi A, Caroff S, Kushner M et al (1987). Regional brain function in schizophrenia: Ii. repeated evaluation with positron emission tomography. Arch Gen Psychiatry 44: 126-129.

Handley R, Zelaya FO, Reinders AATS, Marques TR, Mehta MA, O'Gorman $\mathrm{R}$ et al (2013). Acute effects of single-dose aripiprazole and haloperidol on resting cerebral blood flow $(\mathrm{rCBF})$ in the human brain. Hum Brain Mapp 34: 272-282.

Hietala J, Syvälahti E, Kuoppamäki M, Haaparanta M, Ruotsalainen U, Vuorio K et al (1995). Presynaptic dopamine function in striatum of neuroleptic-naive schizophrenic patients. Lancet 346: 1130-1131.

Hietala J, Syvälahti E, Vilkman H, Vuorio K, Räkköläinen V, Bergman J et al (1999). Depressive symptoms and presynaptic dopamine function in neuroleptic-naive schizophrenia. Schizophr Res 35: 41-50.

Howes OD, Montgomery AJ, Asselin M et al (2009). Elevated striatal dopamine function linked to prodromal signs of schizophrenia. Arch Gen Psychiatry 66: 13-20.

Jokinen P, Helenius H, Rauhala E, Bruck A, Eskola O, Rinne JO (2009). Simple ratio analysis of 18 F-fluorodopa uptake in striatal subregions separates patients with early Parkinson disease from healthy controls. J Nucl Med 50: 893-899.

Kay SR, Flszbein A, Opfer LA (1987). The Positive and Negative Syndrome Scale (PANSS) for Schizophrenia. Schizophr Bull 13: 261-276.

Kinahan PE, Rogers JG (1989). Analytic 3D image reconstruction using all detected events. IEEE Trans Nucl Sci 36: 964-968.

Krimer LS, Muly EC, Williams GV, Goldman-Rakic PS (1998). Dopaminergic regulation of cerebral cortical microcirculation. Nat Neurosci 1: 286-289.

Lahti AC, Holcomb HH, Weiler MA, Medoff DR, Tamminga CA (2003). Functional effects of antipsychotic drugs: comparing clozapine with haloperidol. Biol Psychiatry 53: 601-608.

Lahti AC, Weiler MA, Holcomb HH, Tamminga CA, Cropsey KL (2009a). Modulation of limbic circuitry predicts treatment response to antipsychotic medication: a functional imaging study in schizophrenia. Neuropsychopharmacology 34: 2675-2690.

Lahti AC, Weiler MA, Holcomb HH, Tamminga CA, Cropsey KL (2009b). Modulation of Limbic Circuitry Predicts Treatment Response to Antipsychotic Medication: A Functional Imaging Study in Schizophrenia. Neuropsychopharmacology 34: 2675-2690.

Lahti AC, Weiler MA, Medoff DR, Tamminga CA, Holcomb HH (2005). Functional effects of single dose first- and secondgeneration antipsychotic administration in subjects with schizophrenia. Psychiatry Res 139: 19-30.

Laruelle M, Abi-Dargham A, van Dyck CH, Gil R, D'Souza CD, Erdos J et al (1996). Single photon emission computerized tomography imaging of amphetamine-induced dopamine release in drug-free schizophrenic subjects. Proc Natl Acad Sci USA 93: 9235-9240.
Lee HJ, Weitz AJ, Bernal-Casas D, Duffy BA, Choy M, Kravitz AV et al (2016). Activation of direct and indirect pathway medium spiny neurons drives distinct brain-wide responses. Neuron 91: 412-424.

Lieberman JA, Stroup TS, McEvoy JP, Swartz MS, Rosenheck RA, Perkins DO et al (2005). Effectiveness of antipsychotic drugs in patients with chronic schizophrenia. $N$ Engl $J$ Med 353: 1209-1223.

Lindström LH, Gefvert O, Hagberg G, Lundberg T, Bergström M, Hartvig $\mathrm{P}$ et al (1999). Increased dopamine synthesis rate in medial prefrontal cortex and striatum in schizophrenia indicated by L-( $\beta-11 C)$ DOPA and PET. Biol Psychiatry 46: 681-688.

Livingston MG, Scottish Schizophrenia Research Group (1998). Regional cerebral blood flow in first-episode schizophrenia patients before and after antipsychotic drug treatment. Acta Psychiatr Scand 97: 440-449.

Martinez D, Slifstein M, Broft A, Mawlawi O, Hwang DR, Huang Y et al (2003). Imaging human mesolimbic dopamine transmission with positron emission tomography. Part II: amphetamineinduced dopamine release in the functional subdivisions of the striatum. J Cereb Blood Flow Metab 23: 285-300.

McGowan S, Lawrence AD, Sales T, Quested D, Grasby P (2004). Presynaptic dopaminergic dysfunction in schizophrenia: a positron emission tomographic [18f]fluorodopa study. Arch Gen Psychiatry 61: 134-142.

Meyer-Lindenberg A, Miletich RS, Kohn PD, Esposito G, Carson RE, Quarantelli $M$ et al (2002). Reduced prefrontal activity predicts exaggerated striatal dopaminergic function in schizophrenia. Nat Neurosci 5: 267-271.

Miller DD, Andreasen NC, O'Leary DS, Rezai K, Leonard Watkins G, Boles Ponto LL et al (1997a). Effect of antipsychotics on regional cerebral blood flow measured with positron emission tomography. Neuropsychopharmacology 17: 230-240.

Miller DD, Rezai K, Alliger R, Andreasen NC (1997b). The effect of antipsychotic medication on relative cerebral blood perfusion in schizophrenia: assessment with technetium-99m hexamethylpropyleneamine oxime single photon emission computed tomography. Biol Psychiatry 41: 550-559.

Nozaki S, Kato M, Takano H, Ito H, Takahashi H, Arakawa R et al (2009). Regional dopamine synthesis in patients with schizophrenia using L-[ $\beta-11 C]$ DOPA PET. Schizophr Res 108: 78-84.

Obermeier M, Mayr A, Schennach-Wolff R, Seemuller F, Moller HJ, Riedel M (2010). Should the PANSS be rescaled? Schizophr Bull 36: 455-460.

Patlak CS, Blasberg RG (1985). Graphical evaluation of blood-tobrain transfer constants from multiple-time uptake data. Generalizations. J Cereb Blood Flow Metab 5: 584-590.

Peroutka SJ, Snyder SH (1980). Relationship of neuroleptic drug effects at brain dopamine, serotonin, alpha-adrenergic, and histamine receptors to clinical potency. Am J Psychiatry 137: 1518-1522.

Reith J, Benkelfat C, Sherwin A, Yasuhara Y, Kuwabara H, Andermann F et al (1994). Elevated dopa decarboxylase activity in living brain of patients with psychosis. Proc Natl Acad Sci USA 91: 11651-11654.

Samara MT, Dold M, Gianatsi M, Nikolakopoulou A, Helfer B, Salanti G et al (2016). Efficacy, acceptability, and tolerability of antipsychotics in treatment-resistant schizophrenia: a network meta-analysis. JAMA Psychiatry 73: 199-210.

Seeman P, Tallerico T (1999). Rapid release of antipsychotic drugs from dopamine D2 receptors: an explanation for low receptor occupancy and early clinical relapse upon withdrawal of clozapine or quetiapine. Am J Psychiatry 156: 876-884.

Skirboll LR, Bunney BS (1979). The effects of acute and chronic haloperidol treatment on spontaneously firing neurons in the caudate nucleus of the rat. Life Sci 25: 1419-1433.

Sled JG, Zijdenbos AP, Evans AC (1998). A nonparametric method for automatic correction of intensity nonuniformity in MRI data. IEEE Trans Med Imaging 17: 87-97. 
Sokoloff L (1961). Local cerebral circulation at rest and during altered cerebral activity induced by anesthesia or visual stimulation. In: Kety SS, Elkes J (eds). Regional Neurochemistry. Pergamon: New York. pp 107-117.

Valenti O, Cifelli P, Gill KM, Grace AA (2011). Antipsychotic drugs rapidly induce dopamine neuron depolarization block in a developmental rat model of schizophrenia. J Neurosci 31: 12330-12338.

Victoroff J, Coburn K, Reeve A, Sampson S, Shillcutt S (2014). Pharmacological management of persistent hostility and aggression in persons with schizophrenia spectrum disorders: a systematic review. J Neuropsychiatry Clin Neurosci 26: 283-312.

Viviani R, Graf H, Wiegers M, Abler B (2013). Effects of amisulpride on human resting cerebral perfusion. Psychopharmacology 229: 95-103.

Volkow ND, Brodie JD, Wolf AP, Angrist B, Russell J, Cancro R (1986). Brain metabolism in patients with schizophrenia before and after acute neuroleptic administration. J Neurol Neurosurg Psychiatry 49: 1199-1202.

Wallwork RS, Fortgang R, Hashimoto R, Weinberger DR, Dickinson D (2012). Searching for a consensus five-factor model of the Positive and Negative Syndrome Scale for schizophrenia. Schizophr Res 137: 246-250.

Weinstein JJ, Chohan MO, Slifstein M, Kegeles LS, Moore H, Abi-Dargham A (2017). Pathway-Specific Dopamine Abnormalities in Schizophrenia. Biol Psychiatry 81: 31-42.

Yildiz A, Eryilmaz M, Gungor F, Erkilic M, Karayalcin B (2000). Regional cerebral blood flow in schizophrenia before and after neuroleptic medication. Nucl Med Commun 21: 1113-1118.

Yushkevich PA, Piven J, Hazlett HC, Smith RG, Ho S, Gee JC et al (2006). User-guided 3D active contour segmentation of anatomical structures: significantly improved efficiency and reliability. Neuroimage 31: 1116-1128.

Supplementary Information accompanies the paper on the Neuropsychopharmacology website (http://www.nature.com/npp) 